\title{
Gut Microbiota, Cirrhosis, and Alcohol Regulate Bile Acid Metabolism in the Gut
}

\author{
Jason M. Ridlon ${ }^{a, c}$ Dae-Joong Kang ${ }^{b}$ Phillip B. Hylemon ${ }^{a, c}$ \\ Jasmohan S. Bajaja, b \\ ${ }^{a}$ McGuire VA Medical Center, and ${ }^{b}$ Division of Gastroenterology, Hepatology and Nutrition, and ${ }^{c}$ Department of \\ Microbiology and Immunology, Virginia Commonwealth University, Richmond, Va., USA
}

\section{Key Words}

Alcohol · Bile acid metabolism · Gut microbiota

\begin{abstract}
The understanding of the complex role of the bile acid-gut microbiome axis in health and disease processes is evolving rapidly. Our focus revolves around the interaction of the gut microbiota with liver diseases, especially cirrhosis. The bile acid pool size has recently been shown to be a function of microbial metabolism of bile acid, and regulation of the microbiota by bile acids is important in the development and progression of several liver diseases. Humans produce a large, conjugated hydrophilic bile acid pool, maintained through positive-feedback antagonism of farnesoid X receptor (FXR) in the intestine and liver. Microbes use bile acids, and via FXR signaling this results in a smaller, unconjugated hydrophobic bile acid pool. This equilibrium is critical to maintain health. The challenge is to examine the manifold functions of gut bile acids as modulators of antibiotic, probiotic, and disease progression in cirrhosis, metabolic syndrome, and alcohol use. Recent studies have shown potential mechanisms explaining how perturbations in the microbiome affect bile acid pool size and composition. With advancing liver disease and cirrhosis, there is dysbiosis in the fecal, ileal, and colonic mucosa, in addition to a decrease in bile acid concentration in the intestine due to the liver prob-
\end{abstract}

lems. This results in a dramatic shift toward the Firmicutes, particularly Clostridium cluster XIVa, and increasing production of deoxycholic acid. Alcohol intake speeds up these processes in the subjects with and without cirrhosis without significant FXR feedback. Taken together, these pathways can impact intestinal and systemic inflammation while worsening dysbiosis. The interaction between bile acids, alcohol, cirrhosis, and dysbiosis is an important relationship that influences intestinal and systemic inflammation, which in turn determines progression of the overall disease process. These interactions and the impact of commonly used therapies for liver disease can provide insight into the pathogenesis of inflammation in humans.

(c) 2015 S. Karger AG, Basel

\section{Introduction}

A new concept has emerged in recent years regarding the human body. It is a complex ecosystem constituted on a cellular basis primarily of prokaryotes and archaea whose gene content is estimated to encode $99 \%$ of functional genes [1]. Colonized at birth, or perhaps prior to [2], a series of stochastic events and selection pressures culminate in what is recognized as an 'adult' microbiota by the first year of age [3]. Microbial density in the large bowel reaches an impressive $10^{11}$ cells $\mathrm{cm}^{-3}$. Of the 55

\section{KARGER 125}

(c) 2015 S. Karger AG, Base

0257-2753/15/0333-0338\$39.50/0

E-Mail karger@karger.com

www.karger.com/ddi
Jasmohan S. Bajaj, MD, MS, MBBS

Division of Gastroenterology, Hepatology and Nutrition

Virginia Commonwealth University and McGuire VA Medical Center

1201 Broad Rock Boulevard, Richmond, VA 23249 (USA)

E-Mail jsbajaj@vcu.edu 
bacterial known phyla, selection pressures winnow diversity down to two predominant phyla, the Firmicutes and the Bacteroidetes, in addition to minor representation by Actinobacteria and Proteobacteria [4]. The Bacteroidetes are Gram-negative, non-spore-forming, anaerobic, rodshaped bacteria. The Firmicutes inhabiting the gut are Gram-positive, anaerobic, low $\mathrm{G}+\mathrm{C}$ bacteria. As an example of the taxonomic hierarchy, the secondary bile acid-producing species, Clostridium scindens, is found in the genus Lachnoclostridium, within the family Lachnospiraceae, within the order Clostridiales, within the class Clostridia, within the phylum Firmicutes, and within the kingdom Bacteria and the domain Prokarya. Bile acids represent a major positive or negative selective pressure on the gut microbiome. Bile acids accomplish negative selection pressures directly through antimicrobial properties and indirectly through activation of farnesoid X receptor (FXR)-induced antimicrobial peptide synthesis in the small bowel [5]. Conversely, bile acids elicit positive selection pressures directly through growth stimulation by biotransformations of the steroid nucleus and hydroxy groups that provide a source of or sink for electrons during fermentative metabolism (fig. 1). Bile salt deconjugation is a source of the amino acids taurine and glycine that are known to cause blooms of certain microbial taxa of medical importance [6]. In addition, the production of secondary bile acids by certain gut microbes may represent an important means whereby competitors are excluded given that secondary bile acids are significantly more toxic to certain bacteria than primary bile acids. We will explore here the human experience of the bile acidgut microbiome-liver axis in health and cirrhosis.

\section{Modification of Bile Acids by Microbiota in Healthy Individuals}

Exogenous and endogenous compounds that enter the gut rapidly affect the structure and function of the gut microbiome. Dietary components such as fats can increase the levels of bile acids entering the gut. Work done during the 1970s and 1980s firmly demonstrated that diets rich in animal protein and fat significantly increased total fecal bile acids $[7,8]$. A diet high in animal protein and saturated fat stimulates bile secretion through enhanced signaling by cholecystokinin. Consumption of a Western-style diet (high saturated fat/high simple sugar) shifts the microbiome structure toward bile-resistant members, capable of producing toxic and proinflammatory products from bile salts, namely secondary bile acids

Gut Microbiota, Cirrhosis, and Alcohol

Regulate Bile Acid Metabolism
[9] and hydrogen sulfide from taurine [6]. Studies coupling gnotobiotic mice with metatranscriptomics and metabolomics demonstrate that simple-sugar diets promote degradation of protective host mucin providing greater access of toxic microbial metabolites to the gut epithelium $[10,11]$. Rapid alteration of the gut microbiome through an animal-based diet has been observed to significantly increase fecal bile acid levels and the activity of microbial bile acid modifying enzymes in feces [9]. Plant-based diets have been shown to result in lower fecal bile acid levels compared to animal-based diets [9].

Indeed, bile acid feeding largely reproduces the effects observed in high animal protein and fat diets $[12,13]$. It was observed that Firmicutes expanded at the expense of Bacteroidetes, and there was reduced bacterial diversity and density during cholic acid feeding [12]. Rats fed a 'medium cholic acid diet' (est. $0.98 \mathrm{~mm}$ ) or 'high cholic acid diet' (est. $2.55 \mathrm{~mm}$ ) resulted in shifts in a balanced Firmicutes/Bacteroides ratio of $54.1 \% / 30.7 \%$ to $98.6 \%$ of the microbiome represented by Firmicutes [12]. Bile acids, particularly deoxycholic acid (DCA), are thus a major factor shaping the mammalian gut microbiome.

While the mammalian liver is responsible for producing bile acids in the body, germ-free animals retain a global pool of only primary bile acids [14]. Thus, early studies in germ-free animals demonstrated that gut microbes have evolved biochemical pathways to modify host bile acids. Indeed, host-microbe coevolution is evident as the secondary bile acid, lithocholic acid, is a high-affinity ligand for the vitamin $\mathrm{D}$ receptor, while DCA is a highaffinity activator of TGR-5 [15]. As bile salts transit through the small bowel, populations of bacteria increase from $10^{3} / \mathrm{ml}$ in the duodenum to approaching $10^{11} / \mathrm{ml}$ as bile salts enter the large bowel [16]. Species of Lactobacillus and members of the family Enterobacteriaceae express bile salt hydrolase (BSH) enzymes capable of hydrolyzing bile salts to free taurine and glycine and bile acids [16]. As expected, overexpression of recombinant BSH by Escherichia coli in the small bowel of mice resulted in malabsorption of lipids due presumably to reduced capacity to form mixed micelles [17]. Significant weight loss, reduction in serum cholesterol and triglycerides, and increased $\mathrm{BA}$ synthesis ensues through expression of $\mathrm{BSH}$ relative to BSH-E. coli control [17]. Disruption of mixed micelle formation would lead to significant loss of nutrients into the colon and would be expected to alter the microbiome composition. It may be speculated that subtherapeutic levels of antibiotics fed to agricultural animals may result in weight gain through reduced BSH activity in the small bowel. 


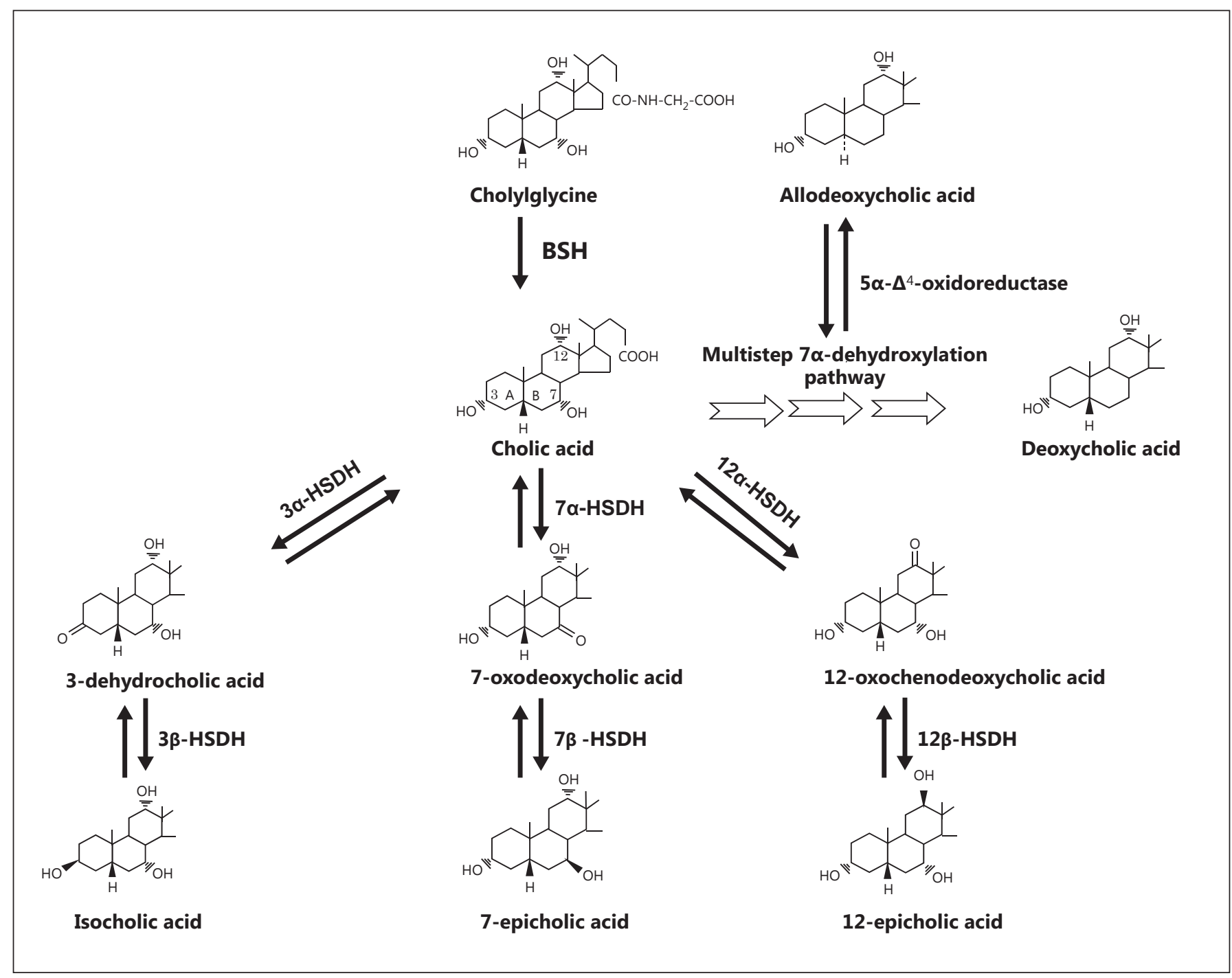

Fig. 1. Bacterial bile salt-biotransforming reactions in the human intestinal tract. The 3-, 7-, and 12-hydroxy group carbons of cholic acid are numbered and the AB rings are identified. Reprinted from Ridlon et al. [16].

In the large bowel, BSH is a redundant feature of the microbiome, and one of significant importance to individual microbes and presumably for the overall microbiome. Functional metagenomic screening of $101 \mathrm{BSH}-$ positive clones from a human fecal sample found that of those with taxonomic information, BSH was located in the three major bacterial divisions Firmicutes (30\%), Bacteroidetes (14.4\%), and Actinobacteria (8.9\%) [18]. Indeed, the only two archaeal species known to inhabit the human gut, Methanobrevibacter smithii and Methanospaera stadtmanae, possess BSH [18]. To date, no BSH inhibitors have been reported, though the effect of inhibiting $\mathrm{BSH}$ on microbiome structure and function would be of significant interest. It is well known for instance that many pathogens [19] and indeed probiotic bacteria require BSH to colonize [20]. The antimicrobial nature of bile salts, particularly glycine-conjugates, suggests that one role of BSH is detoxification [16, 19]. Even if a particular microbial species, or several, lack BSH, they are in a sense 'covered' due to the presence of other species that rapidly hydrolyze bile salts. It may be that natural selection has favored this redundancy of BSH across diverse taxonomic groups to maintain microbiome functional homeostasis in the face of perturbations that may affect one taxonomic group more severely than others [3]. If an important function, such as $\mathrm{BSH}$, is limited to a narrow 
taxonomy, the loss of BSH function may become a perturbation with cascading effects altering microbiome structure/function and thus the host.

In contrast to $\mathrm{BSH}$, the ability to produce secondary bile acids is limited to a few species in the genus Clostridium, Gram-positive members of the Firmicutes [16]. In healthy individuals, their numbers average from $10^{3}$ to $10^{5}$ per gram wet weight. Representing less than $0.025 \%$ of the microbiome by cell density, they are nonetheless capable of converting several hundred milligrams of primary bile acids to secondary bile acids daily. Bile acid $7 a$-dehydroxylation of the human primary bile acids cholic acid and chenodeoxycholic acid proceeds by a complex, multistep pathway culminating in more hydrophobic bile acids, DCA and lithocholic acid, respectively [16]. Lithocholic acid can also be generated from the $7 \beta$-dehydroxylation of ursodeoxycholic acid (UDCA). UDCA can be introduced exogenously via therapies (ursodiol) or endogenously produced by epimerization of the 7a-hydroxy by 7a-hydroxysteroid dehydrogenases $(\mathrm{HSDH})$ and $7 \beta$-dehydrogenases found in single species (Clostridium absonum) or two separate species expressing one or the other HSDH [21]. The evolution of the $7 \beta$-dehydroxylation pathway appears to have arisen by gene-duplication events. There is significant genetic and enzymatic overlap in the bile acid $7 \alpha$-dehydroxylation and the bile acid $7 \beta$-dehydroxylation pathways [16]. Transport (baiG), ligation to coenzyme A (baiB, baiF), oxidation of the $3 a$-hydroxy (baiA), and the reductive arm of the pathway are common to both $7 \alpha$-hydroxy and $7 \beta$-hydroxy bile acids (fig. 2). The two unique steps, oxidation of $\mathrm{C}_{4}-\mathrm{C}_{5}$ and bile acid 7-dehydration, require separate but homologous enzymes. baiE and baiI encode a bile acid $7 \alpha$-dehydratase and putative bile acid $7 \beta$ dehydratase, respectively (fig. 2), are found on the same multi-gene operon, and encode homologous enzymes in the SnoEL family of proteins. baiCD and baiH are also encoded on the same operon and are clearly homologous sharing a high amino acid sequence identity and are both members of the 'Old Yellow' family of proteins [22]. It is suggested that epimerization of chenodeoxycholic acid to UDCA functions to form a less toxic bile acid, and equilibrium constants favor generation of UDCA [23]. Thus, duplication and subsequent divergence of baiE and baiCD toward recognition of $7 \beta$-hydroxy-bile acids would open up an additional niche. Bile acid $7 \alpha-$ dehydroxylating bacteria express a $7 \alpha-\mathrm{HSDH}$, possibly to regulate flux of primary bile acids through the pathway (7-oxo-bile acids cannot be $7 \alpha / \beta$-dehydroxylated), and would be necessary to reduce 7-oxo-bile acids generated

Gut Microbiota, Cirrhosis, and Alcohol Regulate Bile Acid Metabolism from microbial species expressing $7 \alpha / \beta-H S D H$. Removal of the 7-hydroxy group yields a net 2 reduction; bile acids are electron sinks for these fermenting bacteria. However, this small population of microbes has a disproportionate effect on the structure of the microbiome as DCA is 10 times more antimicrobial than CA.

It is currently unclear whether these organisms are capable of persisting without bile acids. If we could specifically inhibit the bile acid $7 \alpha$-dehydroxylation pathway, would these bacteria be outcompeted? What would the structure/function of the microbiome look like if the bile acid $7 a$-dehydroxylation pathway were inhibited? Such inhibitors may be very useful in preventing and/or treating diseases of the gastrointestinal tract such as colon cancer and gallstone disease in certain patients. These questions await development of specific inhibitors.

\section{Cirrhosis, Bile Acids, and the Microbiome}

High concentrations of bile salts (mM) and rapid transit time reduce significant bacterial growth in the small bowel as well as competition with the host for nutrients $[16,19]$. Bile salts also induce the expression of antimicrobial peptides through activation of the FXR, demonstrating an indirect mechanism by which bile salts exert control over the location and community structure of the microbiome in the gut [5]. As a consequence of the endstage liver disease, cirrhosis, there is a significant reduction in bile flow in the intestines. Since bile acid feeding results in a significant shift in the microbiome toward the Clostridiales within the Firmicutes [12], it may be predicted that decreasing bile acid concentrations in the gut might lead to expansion of taxa at the expense of several taxonomic groups within the Firmicutes.

Recent studies have defined changes in the gut microbiome that occur during cirrhosis [24-27]. Beneficial taxa within the Firmicutes such as Lachnospiraceae, Roseburia, Ruminococcaceae and Blautia are underrepresented in cirrhosis [24-27]. Members of these taxa are part of the normal microbiota and are key producers of the beneficial short-chain fatty acid, butyrate. These taxa also include members with bile acid $7 a$-dehydroxylation activity [28]. Indeed, conversion of primary bile acids to secondary bile acids is significantly reduced in cirrhotic patients [29].

Several studies have suggested that members of the oral microbiome occur in the context of cirrhosis, suggesting that bile salts and gastric acid normally exclude oral microbes from colonizing the lower gastrointestinal 


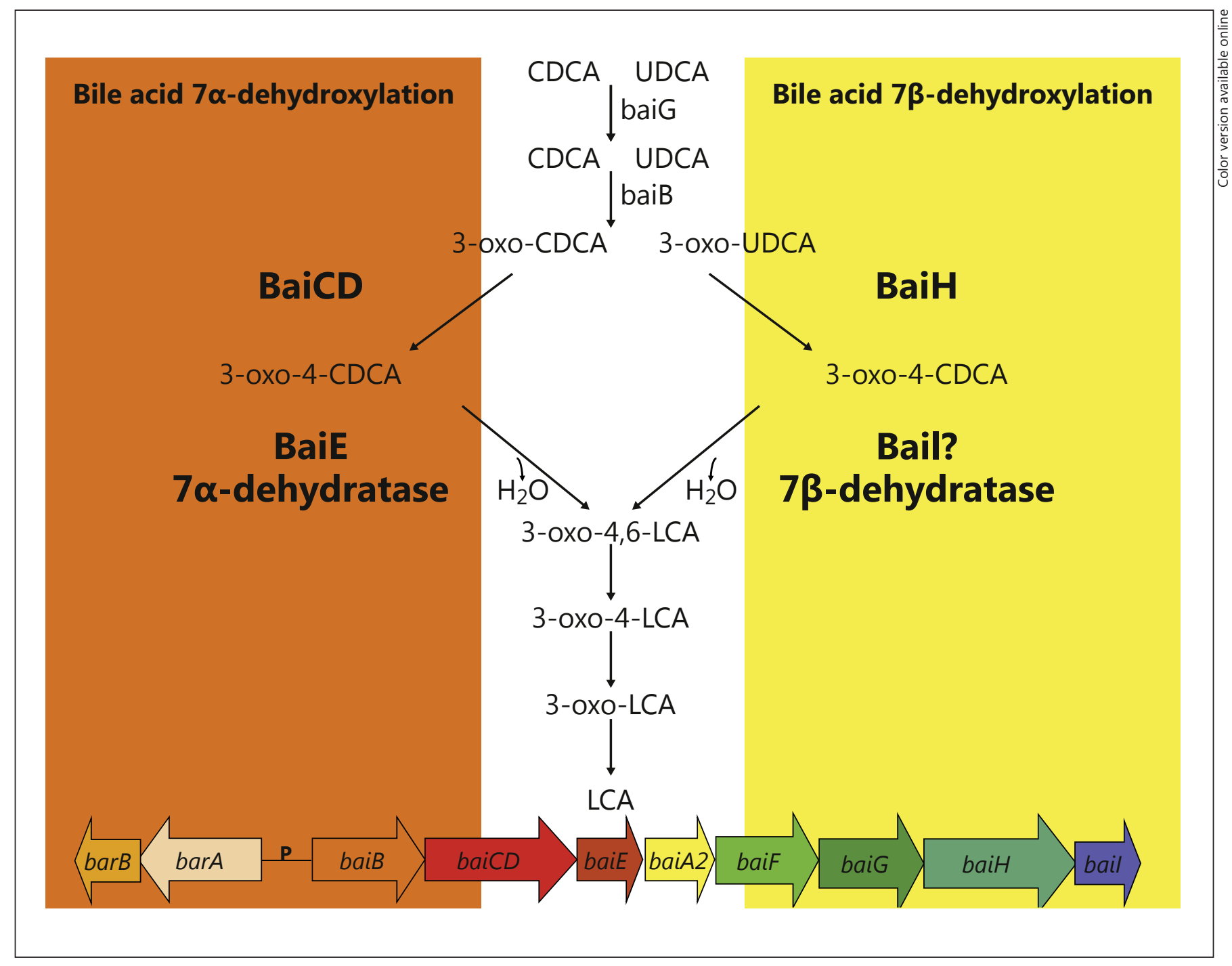

Fig. 2. Common and unique biochemical steps in the bile acid $7 \alpha$-dehydroxylating and $7 \beta$-dehydroxylating pathways in $C$. scindens. Steps unique to bile acid $7 \alpha-\mathrm{DeOH}$ encoded by the baiCD and baiE genes (orange region; colors refer to the online version only). The step unique to $7 \beta-\mathrm{DeOH}$ of UDCA is encoded by the baiH gene (yellow region). The baiI gene is hypothesized to encode bile acid $7 \beta$-dehydratase. Below the pathway is the organization of the bai genes in C. scindens. tract $[25,27]$. Gram-negative members of the Alcaligenaceae, Enterobacteriaceae, and Porphyromonadaceae and Gram-positive members in Streptococcaceae are overrepresented in stool of cirrhotic patients [24-27]. We have previously shown a direct relationship between cognitive impairment (hepatic encephalopathy) in cirrhotic patients and levels of Alcaligenaceae and Porphyromonadaceae [24]. A recent comparison of gut microbial genome content (metagenome) between cirrhotics and controls suggests enrichment for genes involved in am- monia production, $\gamma$-aminobutyric acid (GABA) production, and manganese transport systems, each of which is suggested to play a mechanistic role in cognitive problems associated with liver cirrhosis [27]. Further functional genomic approaches (metatranscriptomics) and metabolomics will be necessary in order to determine the role of these products in disease. Reduced colonic levels of bile acids is hypothesized to play an important role in the dysbiosis characterized by a proinflammatory, toxic gut microbiome (fig. 3) [27-29]. 


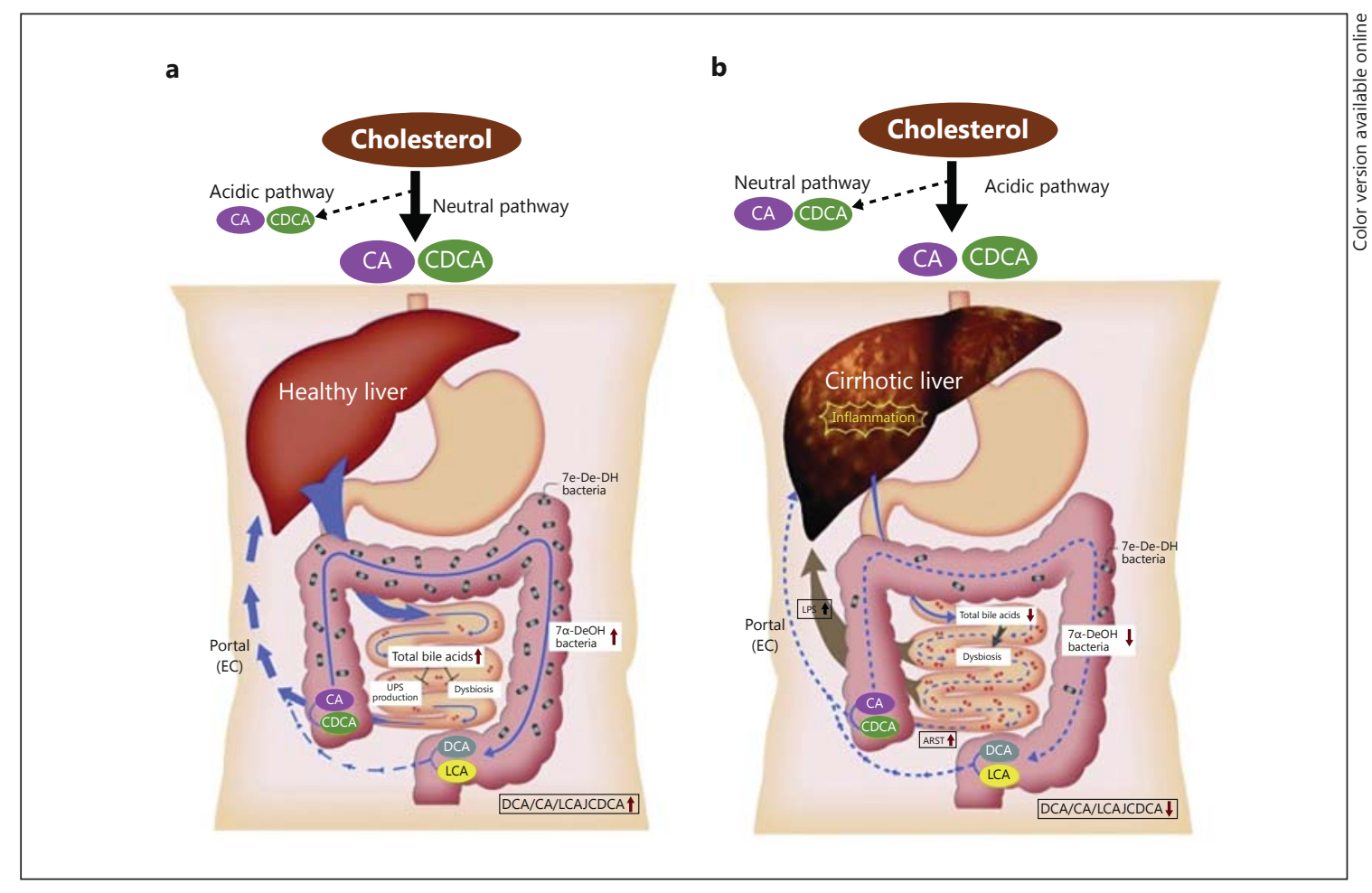

Fig. 3. Proposed model for the relationship between bile acids, the microbiome, and cirrhosis. a In healthy individuals, cholesterol is primarily converted to cholic acid and chenodeoxycholic acid by the neutral bile acid biosynthetic pathway. Sufficient quantities of bile salts enter the small intestine to prevent dysbiosis and the release of inflammatory markers (i.e. lipopolysaccharide). Bile acid $7 \alpha$-dehydroxylating bacteria are found in the normal range $\left(10^{3}-\right.$ $10^{5}$ cells per gram wet weight), and the ratio of secondary to primary bile acids in stool is high. $\mathbf{b}$ In cirrhosis, the neutral pathway is repressed due to downregulation of CYP7A1 by proinflamma-

\section{Effects of Alcohol on Fecal Bile Acid Levels and the Microbiome}

A link has been found between the secondary bile acid DCA, hepatic stellate cells, and hepatocarcinoma in a mouse model of liver cancer [30]. Bile acid products of the gut microbiome may induce inflammation in liver leading to cirrhosis, as stellate cells are active in this process [31]. Alcohol is known to stimulate bile acid synthesis in humans [31]. Recently, it has been reported that alcohol induces bile acid synthesis through activation of hepatic cannabinoid receptor type 1 and CREBH [32]. Kakiyama et al. [33] have shown that cirrhotic patients who are currently drinking have significantly higher levels of fecal secondary bile acids compared to cirrhotic patients abstaining from alcohol ( $>6$ months), nonalcoholic cirrhotics, drinkers tory cytokines, and the acidic pathway is the primary pathway for bile acid synthesis. Dysbiosis occurs due to lower concentration of bile salts entering the small bowel. This dybiosis is characterized by inflammation due to an increase in organisms with potent lipopolysaccharide such as members of Enterobacteriaceae. The population of $7 \alpha$-dehydroxylating bacteria in the colon is hypothesized to decrease due to lower levels of primary bile acids which are thought to serve as an energy source. Consequently, the ratio of secondary/primary bile acids is low in cirrhosis. Reproduced with permission from Gut Microbes [28]. without cirrhosis, and even healthy controls who abstain from alcohol. Cirrhotic patients actively drinking also showed significant increases in mRNA of proinflammatory markers (TNF- $\alpha$, IL-6, IL-1 $\beta$, MCP-1) in colonic but not ileal tissue compared with all other groups [33]. COX2 was also significantly higher in both the ileum and colon of actively drinking cirrhotic patients relative to other groups; however, mRNA levels were highest in the colon [33]. This study by Kakiyama et al. [33] also suggested that alcohol-induced activation of cannabinoid receptor type 1 overrides downregulation of Cyp7A1 by fibroblast growth factor 19. This is supported by increased serum levels of conjugated DCA, and by the finding that mRNA levels of FXR and fibroblast growth factor 19, but not SHP, were increased in the ileum and sigmoid colon of drinkers, but not nonalcoholic cirrhotics or control patients [33]. 
Total fecal bile acids and fecal secondary bile acids in currently drinking alcoholic cirrhotics were 3-4 times the levels of both nonalcoholic and abstinent alcoholic cirrhotic patients ( 8.9 vs. 2.9 vs. $2.29 \mu \mathrm{mol} / \mathrm{g}$, respectively) [33]. DCA has been reported to activate cell-signaling pathways (EGFR, AKT, ERK 1/2, PKC, $\beta$-catenin, Cox-2) and to generate stress through reactive oxygen species (NADPH oxidase, PLA2) and inflammation NF- $\kappa \mathrm{B}$ and proinflammatory cytokine synthesis [34]. Interestingly, the gut microbiome of currently drinking alcoholic cirrhotic patients was largely unchanged with the exception of a significant increase in the Veillonellaceae within the phylum Firmicutes and a significant decrease in Bacteroidaceae and Porphyromonadaceae within the phylum Bacteroidetes [33]. It should be remembered that a major shift occurred in the microbiome of rats fed high levels of CA resulting in members of the Firmicutes dominating at the expense of Gram-negative taxa [12]. However, despite bile acid concentrations in the small and large bowel of human alcoholic cirrhotics surpassing healthy control levels, dysbiosis persisted in cirrhotic patients. Thus, while bile acids are important in shaping the healthy adult microbiome, they are only one factor within a larger context of factors shaping the dysbiosis recently defined in alcoholic cirrhosis.

\section{Probiotics and Secondary Bile Acids}

A recent randomized, double-blind, placebo-controlled study of cirrhotic patients evaluated the efficacy of Lactobacillus rhamnosus GG ATCC strain 53103 (LGG) on cognition, systemic inflammation, fecal bile acids, and gut microbiome composition [35]. Bajaj et al. [35] observed a significant reduction in serum endotoxin and TNF- $\alpha$ in the LGG group only. At baseline, the placebo and LGG treatment group had no significant differences in stool microbiome. However, a significant increase was reported in members of the Firmicutes (Lachnospiraceae and Clostridiales XIV) and a decline in taxa associated with worsening disease and cognitive problems (Enterobacteriaceae and Porphyromonadaceae) in the LGG group, but not the placebo group [35]. A significant increase in DCA was also observed in the placebo group relative to baseline levels [35]. A recent clinical trial achieved similar results through use of the antibiotic rifaximin, which targets bacterial function, and showed improvement in dysbiosis, reduced DCA levels, and reduced endotoxemia [36].

Recent work by Zhang et al. [37] examined the effect of another probiotic bacterium, Lactobacillus casei Zhang, on fecal bile acids and inflammation during a high-fat sucrose (HFS) diet in a model of type 2 diabetes. They reported that blood lipid-lowering effects of $L$. casei Zhang are due to fecal bile acid elimination, supported by a significant reduction of plasma bile acids and a significant increase in total fecal bile acids [37]. However, despite significant increases in bile acid input, secondary bile acid levels were significantly lower in a probiotic + HFS diet relative to an HFS diet alone. qPCR quantification revealed significant increases in the levels of $C$. scindens in an HFS diet relative to a control chow diet and HFS + probiotic diet [37]. A diminution in the levels of $C$. scindens or in general bile acid $7 a$-dehydroxylating activity by $C$. scindens and other species capable of secondary bile acid formation may be responsible for decreased secondary bile acid levels. Decades of research have implicated increased fecal and serum DCA levels with colon cancer, and now liver cancer. Probiotics may thus be an important tool in reducing the levels and activity of bile acid $7 \alpha / \beta$-dehydroxylating bacteria, finding applications in prevention of diseases of the gastrointestinal tract among alcoholics and individuals consuming a high-fat, high-simple-sugar diet.

\section{Conclusions}

The 'omics' revolution and attention to our 'microbial co-conspirators' is leading to new thinking about treating disease in the gastrointestinal tract and beyond. For instance, cognitive problems associated with liver disease are treated by targeting the gut microbiome [25]. This long reach of the human microbiome and the notion that the human body is an ecosystem is underscored by recent applications of systems biology to cirrhosis [25, 26, 29, 33, 36]. Cholestasis appears to generate system inflammation due largely to the absence of adequate bile salt concentrations in the small bowel allowing for colonization of the small bowel by lipopolysaccharide-producing members of the oral microbiota $[27,28]$. Dysbiosis associated with alcohol and cirrhosis of the liver is currently being defined by high-throughput techniques that define community structure and gene content (16s rDNA sequencing, metagenomics), gene expression (metatranscriptomics), and metabolic phenotype (metabolomics). The bile acid pool size and composition is the result of a complex interaction between the three domains of life, Eukarya, Prokarya, and Archaea, which make up the human ecosystem. Defining the role of bile acids in complex human diseases, changes in the composition of bile acids as a result of diet, antibiotics, probiot- 
ics, and other perturbations will require the ability to measure changes in bile acid-modifying microbes, host regulation of synthesis and excretion of bile acids, and how these networks of genes and metabolites are predictably altered. This must necessarily be the goal of 21 st century bile acid-microbiome research.

\section{Disclosure Statement}

There are no disclosures for any author. This work was partly supported by grants VA Merit Review Grant CX001076, NIAAA grant. RO1AA020203 and NIDDK grant RO1DK087913 awarded to Jasmohan S Bajaj.

\section{References}

1 Qin J, Li R, Raes J, et al: A human gut microbial gene catalogue established by metagenomic sequencing. Nature 2009;464:59-65.

-2 Aagaard K, Ma J, Antony KM, et al: The placenta harbors a unique microbiome. Sci Transl Med 2014;6:237ra65.

-3 Ley RE, Peterson DA, Gordon JI: Ecological and evolutionary forces shaping microbial diversity in the human intestine. Cell 2006;124 837-848.

4 Eckburg PB, Bik EM, Berstein CN, et al: Diversity of the human intestinal microbial flora. Science 2005;308:1635-1638.

5 Inagaki T, Moschetta A, Lee Y, et al: Regulation of antibacterial defense in the small intestine by the nuclear bile acid receptor. Proc Natl Acad Sci USA 2006;103:3920-3925.

6 6 Devkota S, Wang Y, Musch MW, et al: Dietary-fat-induced taurocholic acid promotes pathobiont expansion and colitis in $1110^{-/-}$ mice. Nature 2012;487:104-108.

7 Reddy BS: Diet and excretion of bile acids. Cancer Res 1981;41:3766-3768.

8 Reddy BS, Wynder EL: Metabolic epidemiology of colon cancer: fecal bile acids and neutral sterols in colon cancer patients with adenomatous polyps. Cancer 1977;39:25332539.

-9 David LA, Maurice CF, Carmody RN, et al: Diet rapidly and reproducibly alters the human gut microbiome. Nature 2013;505:559563.

10 Sonnenburg JL, Xu J, Leip DD, et al: Glycan foraging in vivo by an intestine-adapted bacterial symbiont. Science 2005;307:1955-1959.

11 Martens EC, Chiang HC, Gordon JI: Mucosal glycan foraging enhances fitness and transmission of a saccharolytic human gut bacterial symbiont. Cell Host Microbe 2008;4:447457.

12 Islam KB, Fukiya S, Hagio M, et al: Bile acid is a host factor that regulates the composition of the cecal microbiota in rats. Gastroenterology 2011;141:1773-1781.

13 Yokota A, Fukiya S, Islam KB, et al: Is bile acid a determinant of the gut microbiota on a high-fat diet? Gut Microbes 2012;3:455-459.
14 Swann JR, Want EJ, Geier FM, et al: Systemic gut microbial modulation of bile acid metabolism in host tissue compartments. Proc Natl Acad Sci USA 2011;108:4523-4530.

15 Makishima M, Lu TT, Xie W, et al: Vitamin D receptor as an intestinal bile acid sensor. Science 2002;296:1313-1316.

16 Ridlon JM, Kang D, Hylemon PB: Bile salt biotransformations by human intestinal bacteria. J Lipid Res 2006;47:241-259.

17 Joyce SA, MacSharry J, Casey PG, et al: Regulation of host weight gain and lipid metabolism by bacterial bile acid modification in the gut. Proc Natl Acad Sci USA 2014;111:74217426.

18 Jones BV, Begley M, Hill C, et al: Functional and comparative metagenomic analysis of bile salt hydrolase activity in the human gut microbiome. Proc Natl Acad Sci USA 2008; 105:13580-13585.

19 Begley M, Gahan CGM, Hill C: The interaction between bacteria and bile. FEMS Microbiol Rev 2005;29:625-651.

20 Begley M, Hill C, Gahan CGM: Bile salt hydrolase activity in probiotics. Appl Environ Microbiol 2006;72:1729-1738.

21 White BA, Fricke RJ, Hylemon PB: $7 \beta$-Dehydroxylation of ursodeoxycholic acid by whole cells and cell extracts of Eubacterium species V.P.I. 12708. J Lipid Res 1982;22: 145-153.

22 Kang D, Ridlon JM, Moore DR 2nd, et al: Clostridium scindens baiCD and baiH genes encode stereo-specific 7alpha/7beta-hydroxy-3-oxo-delta4-cholenoic acid oxidoreductases. Biochim Biophys Acta 2008;1781: 16-25.

23 Macdonald IA, White BA, Hylemon PB: Separation of $7 \alpha$ - and $7 \beta$-hydroxysteroid dehydrogenase activities from Clostridium absonum ATCC\# 27555 and cellular response of this organism to bile acid inducers. J Lipid Res 1983;24:1119-1126.

24 Chen Y, Yang F, Lu H, et al: Characterization of fecal microbial communities in patients with liver cirrhosis. Hepatology 2011;54:562572.

25 Bajaj JS, Ridlon JM, Hylemon PB, et al: Linkage of gut microbiome with cognition in hepatic encephalopathy. Am J Physiol Gastrointest Liver Physiol 2012;302:G168-G175.
26 Bajaj JS, Heuman DM, Hylemon PB, et al: Altered profile of human gut microbiome is associated with cirrhosis and its complications. J Hepatol 2014;60:940-947.

27 Qin N, Yang F, Li A, et al: Alteration of the human gut microbiome in liver cirrhosis. $\mathrm{Na}$ ture 2014;513:59-64.

28 Ridlon JM, Alves JM, Hylemon PB, et al: Cirrhosis, bile acids and gut microbiota: unraveling a complex relationship. Gut Microbes 2013;4:1-6.

29 Kakiyama G, Pandak WM, Gillevet PM, et al Modulation of the fecal bile acid profile by gut microbiota in cirrhosis. J Hepatol 2013;58: 949-955.

30 Yoshimoto S, Loo TM, Atarashi K, et al: Obesity-induced gut microbial metabolite promotes liver cancer through senescence secretome. Nature 2013;499:97-101.

-31 Axelson M, Mork B, Sjovall J: Ethanol has an acute effect on bile acid biosynthesis in man. FEBS Lett 1991;281:155-159.

-32 Chanda D, Kim YH, Li T, et al: Hepatic cannabinoid receptor type 1 mediates alcoholinduced regulation of bile acid enzyme genes expression via CREBH. PLoS One 2013; 8:e68845.

33 Kakiyama G, Hylemon PB, Zhou H, et al: Colonic inflammation and secondary bile acids in alcoholic cirrhosis. Am J Physiol Gastrointest Liver Physiol 2014;306:G929-G937.

34 Hylemon PB, Zhou H, Pandak WM, et al: Bile acids as regulatory molecules. J Lipid Res 2009;50:1509-1520.

35 Bajaj IS, Heuman DM, Hylemon PB, et al: Randomized clinical trial: Lactobacillus GG modulates gut microbiome, metabolome and endotoxemia in patients with cirrhosis. Aliment Pharmacol Ther 2014:39:1113-1125.

36 Bajaj IS, Heuman DM, Sanyal AJ, et al: Modulation of the metabiome by rifaximin in patients with cirrhosis and minimal hepatic encephalopathy. PLoS One 2013;8:e60042.

37 Zhang Y, Guo X, Guo J, et al: Lactobacillus casei reduces susceptibility to type 2 diabetes via microbiota-mediated body chloride ion influx. Sci Rep 2014;4:5654.
Gut Microbiota, Cirrhosis, and Alcohol Regulate Bile Acid Metabolism 